\title{
Capital Cost Assessment of Concentrated Solar Power Plants Based on Supercritical Carbon Dioxide Power Cycles
}

\author{
Francesco Crespi \\ David Sánchez* \\ Tomás Sánchez \\ Department of Energy Engineering, University of Seville \\ Camino de los descubrimientos s/n, 41092 Seville, Spain \\ crespi@us.es, ds@us.es, tms|@us.es \\ Gonzalo S. Martínez \\ AICIA \\ Camino de los descubrimientos s/n, 41092 \\ Seville, Spain \\ gsm@us.es
}

\begin{abstract}
Previous work by the authors has shown that broader analyses than those typically found in literature (in terms of operating pressures allowed) can yield interesting conclusions with respect to the best candidate cycles for certain applications. This has been tested for the thermodynamic performance ( $1^{\text {st }}$ and $2^{\text {nd }}$ Laws) but it can also be applied from an economic standpoint. This second approach is introduced in this work where typical operating conditions for CSP applications (current and future generations of solar tower plants) are considered $\left(750^{\circ} \mathrm{C}\right.$ and $30 \mathrm{MPa}$ ). For these, the techno-economic performance of each cycle is assessed in order to identify the most cost-effective layout when it comes to the Overnight Capital Cost. This analysis accounts for the different contributions to the total cost of the plant, including all the major equipment that is usually found in a CSP power plant such as the solar field and thermal energy storage system. The work is thus aimed at providing guidelines to professionals in the area of basic engineering and pre-feasibility study of CSP plants who find themselves in the process of selecting a particular power cycle for a new project (set of specifications and boundary conditions).
\end{abstract}

\section{NOMENCLATURE \\ $s \mathrm{CO}_{2} \quad$ Supercritical Carbon Dioxide. \\ TIT Turbine Inlet Temperature $\left[{ }^{\circ} \mathrm{C}\right]$. \\ CSP Concentrated Solar Power.}

${ }^{*}$ Corresponding author. Email: $\underline{\text { ds@ } @ \text { us.es }}$ 


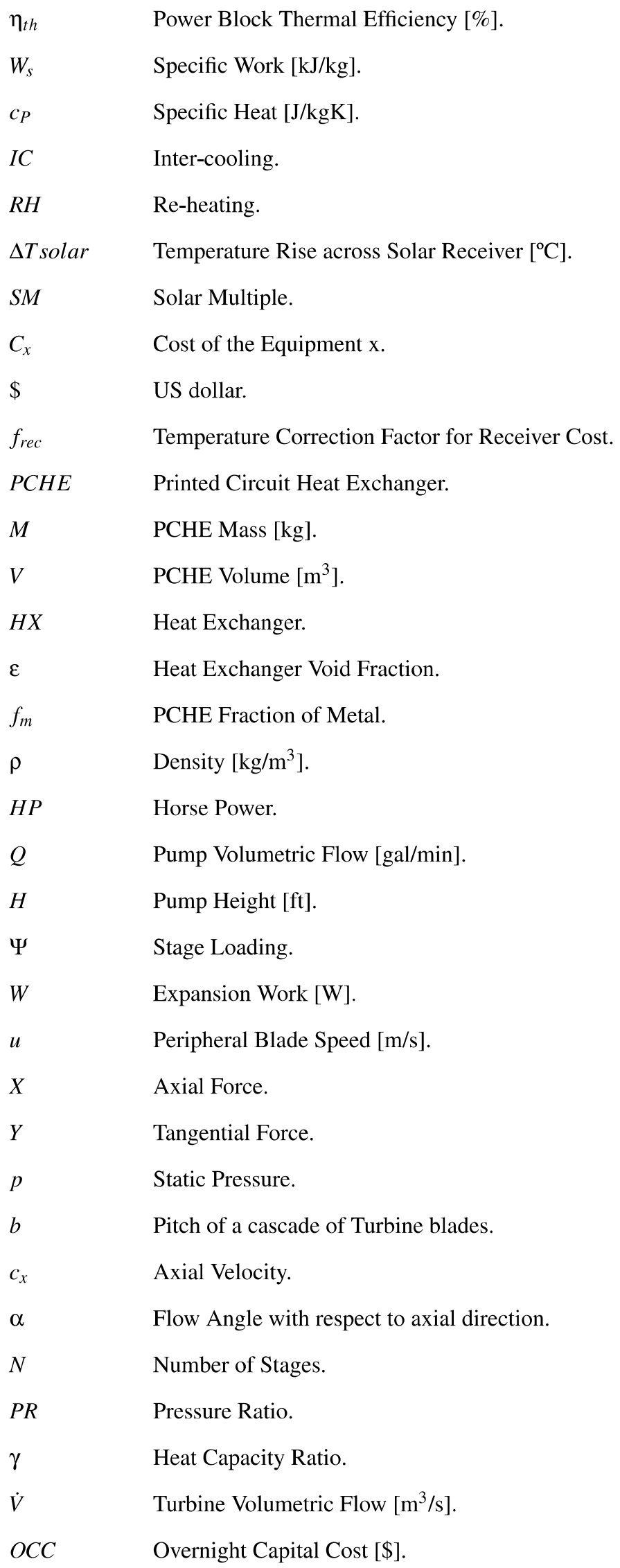




$\begin{array}{ll}\text { Subscripts } & \\ s & \text { Molten Salt. } \\ S F & \text { Solar Field. } \\ R & \text { Solar Receiver. } \\ T E S & \text { Thermal Energy Storage. } \\ r e f & \text { Reference case. } \\ c & \text { PCHE Channel. } \\ m & \text { Metal. } \\ r a w & \text { HX Raw Material. } \\ w & \text { Water. } \\ 1 & \text { Blade row inlet. } \\ 2 & \text { Blade row outlet. } \\ T F X & \text { Thermoflex. } \\ C T & \text { Cooling Tower. } \\ B o P & \text { Balance of Plant. }\end{array}$

\section{INTRODUCTION}

Supercritical Carbon Dioxide power cycles have gained popularity in the last decade exponentially, thanks to the increasing interest of several companies and research institutes worldwide. Nowadays, the thermodynamic potential of this technology is undoubted, as it is also its adaptability to different fields of application, in particular Concentrated Solar Power systems $[1,2]$. There are numerous technical analyses of these cycles, either from a thermodynamic standpoint or integrated into particular applications or energy sources, and most of them have been reviewed by the authors in [3]. In contrast, there is still a great deal of uncertainty when it comes to the economic competitiveness of the technology. Some authors have tried to estimate the cost of $\mathrm{sCO}_{2}$ power cycles, from the fundamental work by Dostal [4] to contemporary works by NETL [5], SuperCritical Technologies Inc. [6] and the Massachusetts Institute of Technology [7]. More specifically, super-alloys for advanced power systems are studied by De Barbadillo [8] and Cich [9] while the cost of Printed Circuit Heat Exchangers is assessed by Hinze [10] and Kim [11]. Regarding CSP, NREL's Solar Advisory Model SAM [12] is the most common tool employed in literature for techno-economic analysis, also using $\mathrm{sCO}_{2}$ cycles [13].

The authors of this paper have developed a systematic analysis of the fundamentals of $\mathrm{sCO}_{2}$ cycles in the last years. A thorough revision of the current state of the art compared more than forty cycle layouts [3], twelve of which were later considered for the in-depth analysis of their thermodynamic performance [14]. This latter work disregarded any technical constraint in terms of working pressures and temperatures of the components, with the aim to assess the inherent thermodynamic potential of each cycle beyond the current state of the art of the components. The figures of merit considered in the assessment were thermal efficiency $\left(\eta_{t h}\right)$, specific work $\left(W_{s}\right)$ and Carnot Factor, hence combining the First and Second Laws of Thermodynamics. For the sake of completeness, four different Turbine Inlet Temperatures were considered (550, 
750,950 and $1150^{\circ} \mathrm{C}$ ), confirming the adaptability of $\mathrm{sCO}_{2}$ technology to various fields of application. Figure 1 shows these results for the cycles in Fig. 2 when operating at $950^{\circ} \mathrm{C}$.

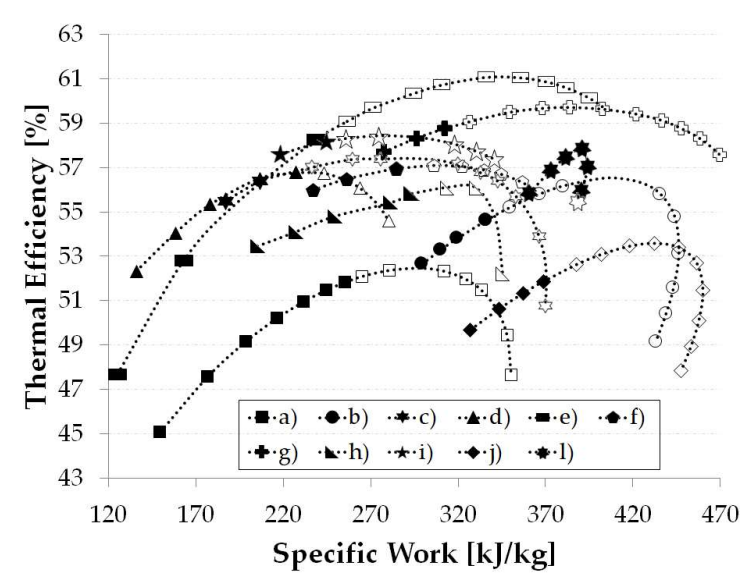

(a) Thermal efficiency against Specific Work.

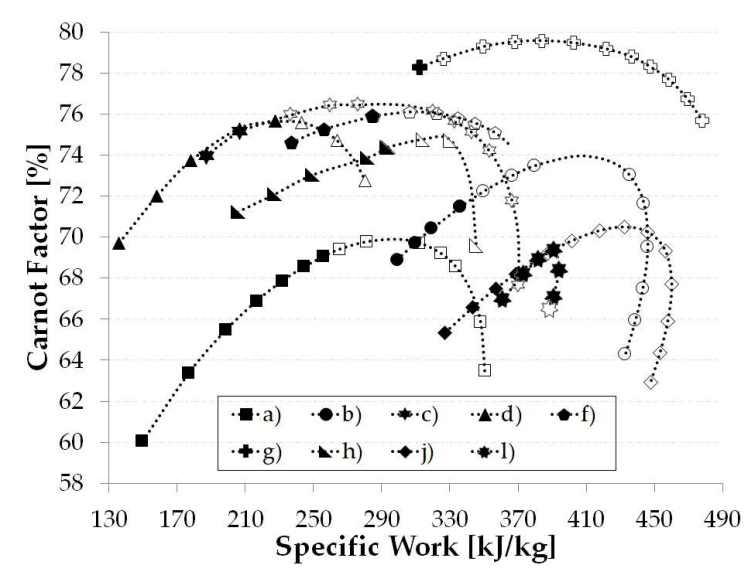

(b) Carnot Factor against Specific Work. Maximum recuperator temperature is limited to $800^{\circ} \mathrm{C}$.

Fig. 1. First and Second Law comparison of $\mathrm{sCO}_{2}$ cycles operating at $950^{\circ} \mathrm{C}$, adapted from [14]. Legend refers to Fig. 2.

It becomes visible that, as long as the $1^{\text {st }}$ Law is concerned $\left(\eta_{t h}\right)$, the Recompression $+I C+R H$, Double Reheated, Partial Cooling $+R H$ and Quasi-Combined cycles are the most interesting. Nevertheless, the scenario changes substantially if the maximum recuperator temperature is limited to $800^{\circ} \mathrm{C}$ and the analysis is based on the $2^{\text {nd }}$ Law. In such case, both the Recompression $+I C+R H$ and Double Reheated layouts are no longer feasible and the Quasi-Combined cycle shifts down dramatically. Globally, a compromise between the three figures of merit confirms that the Partial Cooling $+R H$ cycle is the best choice at this temperature level, followed by the Precompression, Recompression and Partial Cooling layouts.

This systematic thermodynamic approach has recently been complemented with economic features applicable to Concentrated Solar Power plants in [15], following the path set forth by other authors recently [1,2, 16, 17]. Focusing on the costs of Solar Field and Thermal Energy Storage system, the authors have credited that the characteristics of these systems are strongly affected by the operating conditions of the working cycle through $\eta_{t h}$ and the temperature rise of molten salts across the solar receiver $\left(\Delta T_{\text {solar }}\right)$. In the present paper, the cost estimation of these items, performed with SAM and with 


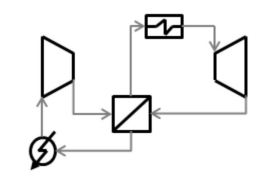

a) Simple Recuperated

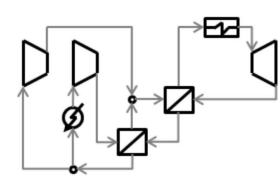

d) Recompression

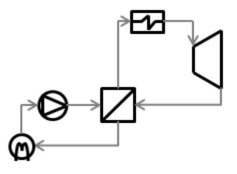

b) Transcritical CO2

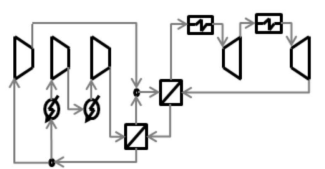

e) Recompression $+\mathrm{RH}+\mathrm{IC}$

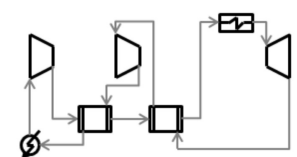

c) Precompression

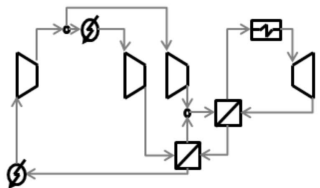

f) Partial Cooling

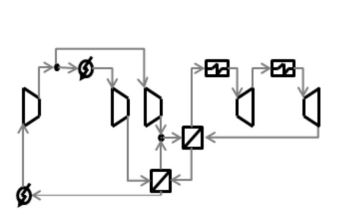

g) Partial Cooling $+\mathrm{RH}$

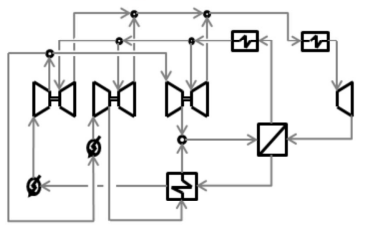

h) Schroder-Turner

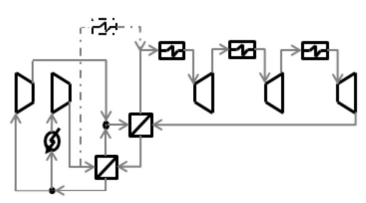

i) Double Reheated
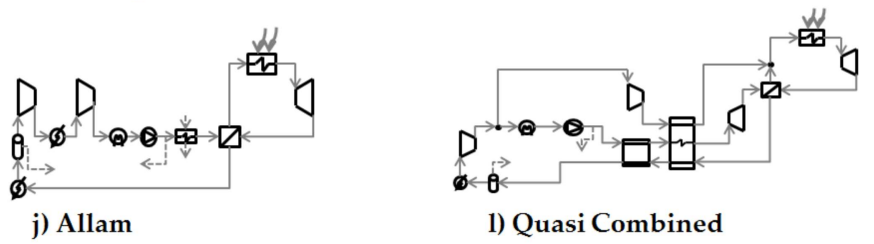

Fig. 2. Summary of cycle layouts. Adapted from [14].

an in-house model respectively, has been combined with the already existing methodology for the thermodynamic analysis. Then, the resulting tool has been upgraded with uncertainty quantification in regards to the economic input dataset, which is a unique feature with respect to other works in literature.

The specifications of the reference power plant used in these analyses are summarised in Table 1.

\begin{tabular}{ccccccc}
\hline \hline $\begin{array}{c}\text { Power Output } \\
{\left[\mathrm{MW}_{e l}\right]}\end{array}$ & $\begin{array}{c}\mathrm{P}_{\max , 5 \mathrm{CO}_{2}} \\
{[\mathrm{MPa}]}\end{array}$ & $\begin{array}{c}\mathrm{TIT} \\
{\left[{ }^{\circ} \mathrm{C}\right]}\end{array}$ & $\begin{array}{c}\mathrm{T}_{s, \min } \\
{\left[{ }^{\circ} \mathrm{C}\right]}\end{array}$ & $\begin{array}{c}\mathrm{T}_{s, \text { max }} \\
{\left[{ }^{\circ} \mathrm{C}\right]}\end{array}$ & $\begin{array}{c}\mathrm{TES}_{\text {capacity }} \\
{[\text { hour }]}\end{array}$ & $\begin{array}{c}\mathrm{SM} \\
{[-]}\end{array}$ \\
\hline 50 & 30 & 750 & 480 & 770 & 10 & 2.4 \\
\hline \hline
\end{tabular}

Table 1. Specifications of the reference power plant.

The work in this paper confirms that $\mathrm{SCO}_{2}$ can potentially be installed at a cost that is comparable with current steam turbine technology. Bearing in mind that the latter technology does not hold the potential to become much more efficient than it currently is, this is a promising result that will be confirmed by Levelised Cost of Electricity calculations in the near future.

The structure of the paper is as follows. First, the cost model is presented, describing the functions estimating the cost of major equipment. Then, the main assumptions behind the Montecarlo analysis for uncertainty quantification are discussed. The last part of the paper provides a discussion of the main results. 


\section{OVERNIGHT CAPITAL COST ESTIMATION}

Estimating the cost of a Concentrated Solar Power plant can become an extremely complex task, depending on the level of detail required [18]. The difficulty lies on the lack of reliable data since these are mostly proprietary, especially when an emergent technology like $\mathrm{sCO}_{2}$ is involved. Accordingly, the cost estimates provided in this work focus on the major components (solar field, thermal energy storage system, heat exchangers and turbomachinery) and Balance of Plant equipment. Once these cost estimators are developed, different levels of uncertainty will be assigned to each input dataset to account for the development stage of each iteam.

\section{Solar Field}

The cost of the solar field $\left(C_{S F}\right)$, calculated with SAM, is very sensitive to the efficiency of the power block as this figure drastically affects the heat input needed for a given power output. This is shown in Fig. 3 where the inverse, non-linear dependence of $C_{S F}$ upon $\eta_{t h}$ is observed [15]. These results correspond to the default Solar Multiple reported in Table 1.

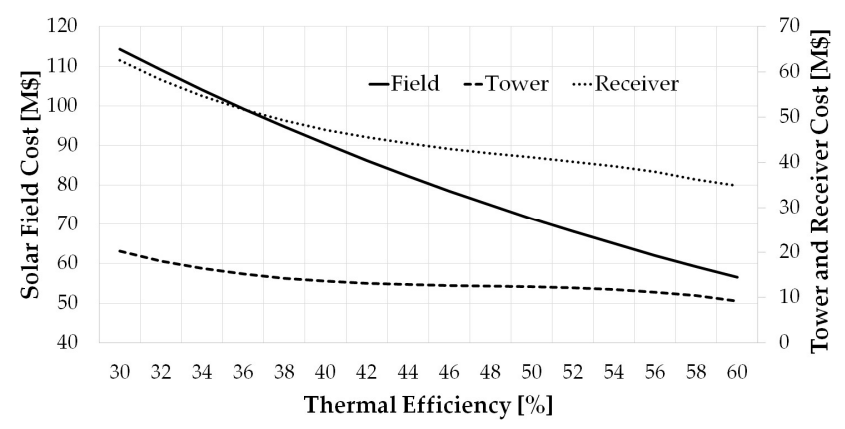

Fig. 3. Solar Subsystem Cost function as produced by SAM. Field Cost on left axis, Tower and Receiver on right axis.

\section{Solar Tower and Receiver}

The cost of these components is again obtained with $S A M$ based on the values given for a reference power plant using steam turbine technology. For the tower, the cost depends on its height which is itself dependent on the thermal efficiency of the power block through heat input [18], Fig. 3. This is the only dependence of the solar tower cost function as no differences are expected between towers in power plants based on steam turbines or $\mathrm{sCO}_{2}$ cycles.

With respect to the receiver, two correction factors have been applied to the reference values calculated with SAM for state-of-the-art molten salts used in contemporary CSP plants. The first correction accounts for the different operating temperature of the receiver, which in this paper is increased to $770{ }^{\circ} \mathrm{C}$ with respect to standard steam technology. In accordance to this, an in order to account for the higher technical risk, a $30 \%$ higher cost is considered regardless of the size of the receiver. The second correction factor takes into account that the working fluid in a CSP plant based on steam turbines and in a plant based on $\mathrm{SCO}_{2}$ technology are likely to exhibit a very different temperature rise across the receiver. This translates into an inversely proportional variation of molten salt flow rate and, therefore, receiver volume. The correction factor takes into account the different energy absorption capacity of state-of-the-art salts used in contemporary CSP plants with respect to 
a high temperature salt like $F L i N a K$, which is the working fluid of choice in this work. FLiNaK is a ternary eutectic alkaline metal fluoride salt mixture characterised by an extremely high degradation temperature (almost $1570{ }^{\circ} \mathrm{C}$ ), whose thermophysical properties are computed with correlations taken from [19]. The resulting correction factor is shown in Eq.(1) where subscripts FLiNaK and ref refer to the said high temperature salt and the solar salt used by default in commercial CSP plants. The cost function of the reference receiver $C_{R, r e f}$ is shown in Fig. 3.

$$
C_{R, F L i N a K}=f_{r e c} \cdot C_{R, r e f}=1.3 \cdot \frac{\bar{c}_{p, r e f} \cdot \Delta T_{r e f}}{\bar{c}_{p, F L i N a K} \cdot \Delta T_{F L i N a K}} \cdot C_{R, r e f}
$$

\section{Thermal Energy Storage System}

The cost of the Thermal Energy Storage system $\left(C_{T E S}\right)$ is calculated with an in-house code whose main characteristics have already been described in [20]. It includes the costs of the inventory of molten salts, the tanks and all the auxiliary equipment needed. The model was originally used for standard CSP plants based on steam turbines operating at $550{ }^{\circ} \mathrm{C}$ but it has been modified for its application to higher operating temperatures. In this work, the maximum operating temperature of the molten salts is set to $770{ }^{\circ} \mathrm{C}$ whereas the minimum temperature is dictated by the working $\mathrm{sCO}_{2}$ cycle, meaning that it has a different value for each configuration. The gap between these two temperatures is the temperature rise across the solar receiver $\left(\Delta T_{\text {solar }}\right)$ which is found to affect the size and cost of the TES largely; this was also the case for the receiver in the previous section. The impact of $\Delta T_{\text {solar }}$ and $\eta_{t h}$ on $C_{T E S}$ has already been explored by the authors in [15] and is shown in Fig. 4.

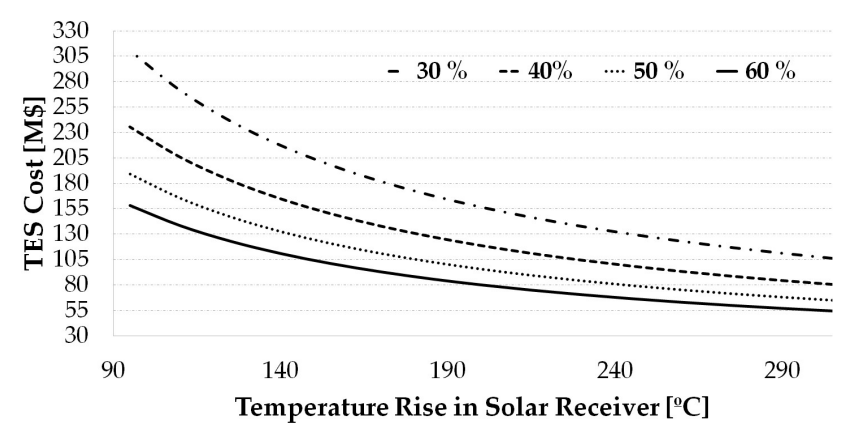

Fig. 4. Cost of Thermal Energy Storage system. Adapted from [15].

\section{Heat Exchangers}

Following the most usual approach, and even if the authors acknowledge that it might not be the best option for a large scale, commercial power plant, Printed Circuit Heat Exchangers (PCHE) have been considered the technology of choice in this analysis. The thermal performance of these equipment has been modelled with an in-house code whose description and validation are provided in $[21,22]$. This code has been improved with new thermal correlations for wavy channels PCHE [11] and a mechanical stress study based on the work by Yoon [23]. To this latter aim, a maximum allowable mechanical stress has 
been set, corresponding to a maximum pressure difference between the hot and cold sides, after which the geometry of the PCHE (channel pitch and wall thickness) is modified to ensure mechanical integrity at the working pressure and temperature of the heat exchanger. This approach links the working pressure and void fraction of the equipment, yielding bulkier PCHEs at higher pressures.

The cost assessment of the PCHE designed with the methodology presented in the foregoing paragraph has then been performed with a methodology based on the works by Dostal [4] and Kim [11]. In these, the mass of the heat exchanger $M$ is obtained from its volume $V$ and void fraction -or porosity- $\left(\varepsilon_{H X}=1-f_{m}\right)$ which is given a reference value taken from literature. The latter parameter represents a sort of "density" of the HX, as defined in Eq.(2) where $D_{c}, P_{c}$ and $t$ are the channel diameter, pitch and plate thickness. The former is set to $3 \mathrm{~mm}$ whilst the other two parameters result from the mechanical analysis (typical values are 0.2 and $0.5 \mathrm{~mm}$ respectively). The total mass is finally obtained by merely multiplying $V, f_{m}$ and $\rho_{m}$ (density of the raw material considered). The cost of the PCHE is then calculated from the cost of the raw material $\left(C_{\text {raw }}\right)$, expressed in $\$ / \mathrm{kg}$, Eq.(3).

$$
f_{m}=1-\frac{\pi \cdot D_{c}^{2}}{8 \cdot P_{c} \cdot t_{c}} \quad ; \quad M=\rho_{m} \cdot V \cdot f_{m}
$$

$$
C_{H X}[\$]=M \cdot C_{\text {raw }}
$$

Two different alloys are considered depending on the operating temperature. Stainless Steel $316 \mathrm{~L}$ is used for the coolers, which do not have to withstand high temperatures, and the recuperators whose maximum temperature does not exceed 475 ${ }^{\circ} \mathrm{C}$. Inconel 617 is employed in those HXs operating at higher temperatures, including the heaters. The maximum allowable mechanical stresses of these alloys, taken from [24,25], are represented as a function of temperature in Fig. 5.

Estimating the manufacturing/processing cost to be added to the raw material (usually supplied in bars) to calculate $C_{\text {raw }}$ in PCHEs is challenging, inasmuch as this information is proprietary of the original equipment manufacturers. This is why the approach presented by Kim et al. to produce a correction factor that could be applied to the un-processed (raw material) cost is used [11]. These authors consider a cost of $150 \$ / \mathrm{kg}$ for the processed Alloy $800 \mathrm{HT}$, which is six times higher than the cost of the un-processed material in the market (ca. $25 \$ / \mathrm{kg}$ ). Applying this correction factor to the aforelisted alloys yields the following cost ranges: Stainless Steel $316 L$ from 20 to $25 \$ / \mathrm{kg}$, Inconel 617 from 120 to $180 \$ / \mathrm{kg}$.

\section{Turbomachinery}

Turbomachinery components are simulated with simple lumped volume models given that these are intended for ondesign performance only [14]. Then, due to the lack of reliable cost data for $\mathrm{sCO}_{2}$ turbomachinery, standard cost estimates for air compressors and centrifugal pumps are employed, as presented in [26]. The cost of centrifugal compressors is obtained 


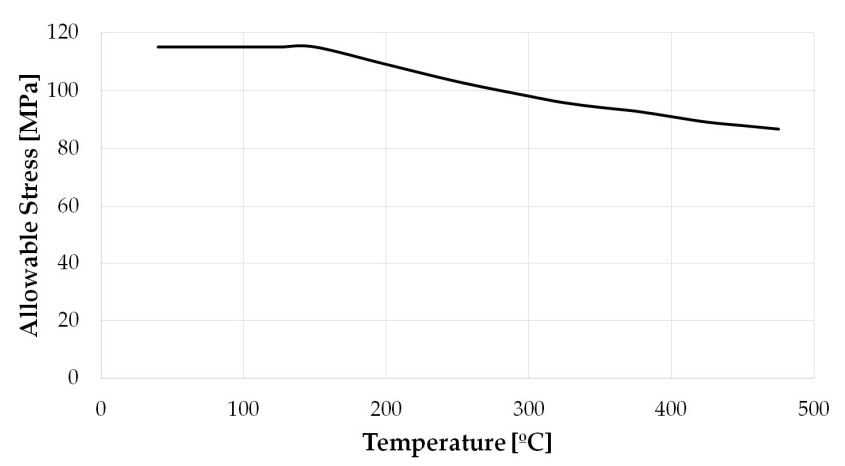

(a) Allowable stress for Stainless Steel 316L (obtained from [24]).

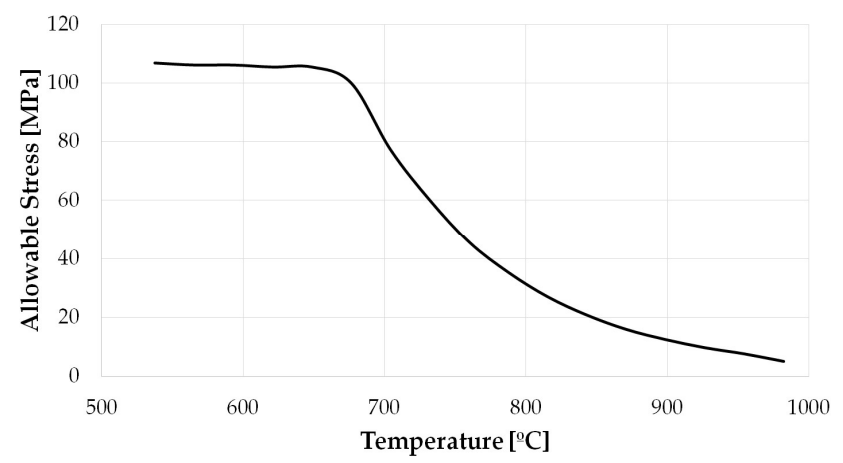

(b) Allowable stress for Inconel617 (obtained from [25])

Fig. 5. Maximum allowable mechanical stresses of material employed in $\mathrm{HX}$ design, as a function of temperature

as a function of the required electric power in Horse Power, Eq.(4), whereas the cost of the pumps is calculated as a function of their volumetric flow $Q$ and head $H$ in $\mathrm{gal} / \mathrm{min}$ and $\mathrm{ft}$, Eq.(5).

$$
\begin{gathered}
C_{\text {compr }}[k \$]=7.90 \cdot \dot{W}_{e l}^{0.62}, \quad 200<\dot{W}_{e l}[H P]<30000 \\
\left\{\begin{array}{l}
C_{\text {pump }}[\$]=2 \cdot F_{T} \cdot C_{b} \\
F_{T}=\exp \left(9.8849-1.6164 \cdot \ln (Q \cdot \sqrt{H})+0.083 \cdot(\ln (Q \cdot \sqrt{H}))^{2}\right) \\
C_{b}=3 \cdot \exp \left(8.833-0.6019 \cdot \ln (Q \cdot \sqrt{H})+0.0519(\ln (Q \cdot \sqrt{H}))^{2}\right)
\end{array}\right.
\end{gathered}
$$

Supercritical $\mathrm{CO}_{2}$ turbines are expected to be less costly than steam turbines of similar output due to the lower footprint brought about by the lower specific volume of the working fluid and the lower pressure ratio of the working cycle (fewer number of stages) [4]. Based on this rationale, the cost of $\mathrm{sCO}_{2}$ turbines is extrapolated from the cost of supercritical steam turbines without steam bleeds, as produced by Thermoflex software [27]. Two correction factors are then applied, the first of which is the ratio of volume flow rate between the reference and $\mathrm{sCO}_{2}$ turbines. The second correction factor is based on the assumption that, due to material strength limitations, stage loading in a $\mathrm{sCO}_{2}$ turbine is roughly $25-30 \%$ lower than in a 
steam turbine, Eq.(6),

$$
\Psi_{\mathrm{sCO}_{2}}=\frac{W_{\text {stage }, \mathrm{SCO}_{2}}}{u_{\mathrm{sCO}}^{2}}=0.75 \cdot \Psi_{\text {steam }}=\frac{W_{\text {stage }, \text { steam }}}{u_{\text {steam }}^{2}}
$$

where $\Psi$ is the stage loading coefficient, $W_{\text {stage,steam }}$ is the expansion work and $u$ is the peripheral blade speed at mean turbine radius. Such a statement can be easily deduced from the following expression of the forces exerted by turbine blades on an incompressible, inviscid flow expanding across a bi-dimensional cascade, given by mass and momentum conservation:

$$
\left\{\begin{array}{l}
F_{x}=\left(p_{1}-p_{2}\right) \cdot b \\
F_{y}=\rho \cdot b \cdot c_{x}^{2} \cdot\left(\tan \alpha_{1}-\tan \alpha_{2}\right)
\end{array}\right.
$$

where $\rho$ is density, $b$ is pitch of the cascade, $c_{x}$ is axial velocity and $\alpha$ is flow angle with respect to the axial direction. The boundary conditions and forces are illustrated in Fig. 6.

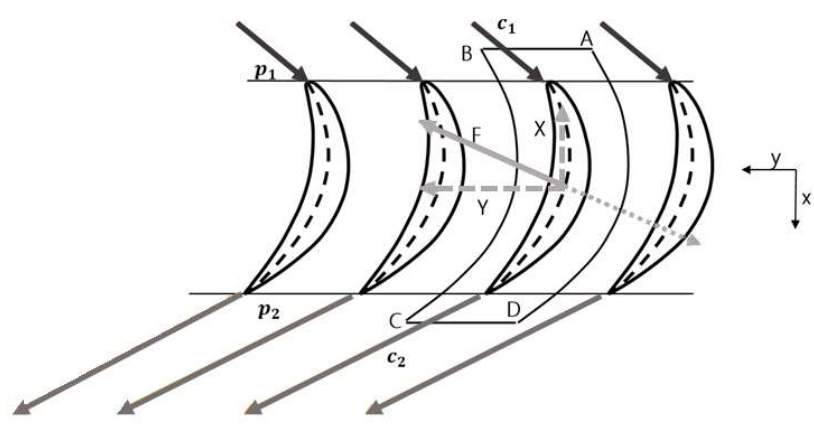

Fig. 6. Forces on a cascade of turbine blades.

Should the steam and carbon dioxide flows turn a similar angle across the cascade (deflection) in Fig. 6, the tangential force $F_{y}$ exerted on the blade would increase proportionally to the change in density and axial velocity squared, Eq.(7). With this in mind, the following observations are noteworthy:

-Turbine inlet density almost doubles when using supercritical carbon dioxide in a CSP application at $750^{\circ} \mathrm{C}$ versus a similar plant using supercritical steam turbines at the standard temperature of $560^{\circ} \mathrm{C}^{1}$

-In addition, the density drop along the expansion line in a supercritical steam cycle is much larger than in a supercritical $\mathrm{CO}_{2}$ cycle due to (i) the much larger expansion ratio of the former cycle and (ii) the higher isentropic exponent of steam. As a result, the average densities of steam and carbon dioxide in these turbines are 40 and $90 \mathrm{~kg} / \mathrm{m}^{3,2}$. The cumulative effect on tangential force, based on these average densities, would be a double $F_{y}$ for $\mathrm{CO}_{2}$.

\footnotetext{
${ }^{1}$ The approximate density of steam at 250 bar and $560^{\circ} \mathrm{C}$ is $75 \mathrm{~kg} / \mathrm{m}^{3}$ whilst carbon dioxide at 300 bar and $750^{\circ} \mathrm{C}$ has a density of $145 \mathrm{~kg} / \mathrm{m}^{3}$.

${ }^{2}$ These densities are based on isentropic expansions from the conditions in footnote ${ }^{1}$ to 0.080 and 75 bar for steam and carbon dioxide respectively GTP-18-1741, Sánchez, Page 10 Copyright (C) 2018 by ASME
} 
- On the other hand, the change in axial velocity can be correlated to the change in speed of sound, which is in the order of $20 \%\left(525 \mathrm{~m} / \mathrm{s}\right.$ for $\mathrm{CO}_{2}$ and $650 \mathrm{~m} / \mathrm{s}$ for steam at turbine inlet). This means a $35 \%$ lower axial velocity squared, hence tangential force, for $\mathrm{CO}_{2}$.

The higher tangential force in a $\mathrm{CO}_{2}$ turbine could be reduced by simply reducing the pitch/chord ratio of the blade

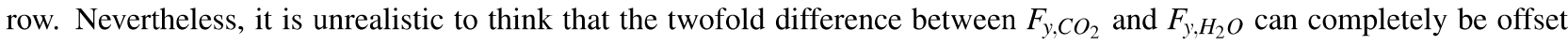
through this effect as this would drastically increase profile losses (friction on the blade passage walls). This is why a 15-25\% lower load coefficient for $\mathrm{sCO}_{2}$ is assumed in Eq.(6), the remainder reduction of $\mathrm{F}_{y, \mathrm{CO}_{2}}$ (if any) relying on a higher solidity. If expansion work in the Rankine steam cycle and in each $\mathrm{sCO}_{2}$ cycle is then expressed as a function of expansion ratio, turbine inlet conditions and properties of the working fluid, the following correction factor can be devised under the assumption that all stages in the turbine exchange equal work

$$
\frac{N_{s \mathrm{CO}_{2}}}{N_{\text {steam }}}=1.5 \cdot \frac{\bar{c}_{p, s \mathrm{CO}} \cdot T I T \cdot\left(1-P R^{\frac{1-\gamma}{\gamma}}\right)}{\Delta h_{\text {steam }}}
$$

where $N$ is the number of stages, TIT and $P R$ are the turbine inlet temperature and pressure ratio of the $\mathrm{sCO}_{2} \mathrm{cycle}_{\mathrm{c}} \mathrm{and}$ $\Delta h_{\text {steam }}$ is the isentropic enthalpy change across the steam turbine. With this information, the resulting cost function of the $\mathrm{sCO}_{2}$ turbine is

$$
C_{\text {turb }}[k \$]=C_{T F X} \cdot \frac{\dot{V}_{\text {sCO }}}{\dot{V}_{\text {steam }}} \cdot \frac{N_{\text {s } \mathrm{CO}_{2}}}{N_{\text {steam }}}
$$

where $C_{T F X}$ is the cost estimate of a supercritical steam turbine of given volumetric flow rate $\dot{V}_{\text {steam }}$ as provided by Thermoflex.

\section{Cooling Tower}

The cost of the cooling tower is also obtained with Thermoflex. To this aim, the inlet temperature of hot water entering the cooling tower and the tower range $\left(\Delta T_{\text {range }}\right)$ are set to $26.8{ }^{\circ} \mathrm{C}$ and $10{ }^{\circ} \mathrm{C}$ respectively. With this information, the water mass flow rate of the cooling tower is easily calculated by merely applying energy conservation with ISO ambient conditions: $15^{\circ} \mathrm{C}, 1 \mathrm{~atm}$ and $60 \% \mathrm{RH}$. The correlation estimating the cost of the cooling tower as a function of the water mass flow rate 
$\dot{m}_{w}$ is presented in Eq.(10).

$$
\left\{\begin{array}{l}
C_{C T}[k \$]=a \cdot \dot{m}_{w}^{6}-b \cdot \dot{m}_{w}^{5}+c \cdot \dot{m}_{w}^{4}+d \cdot \dot{m}_{w}^{3}+e \cdot \dot{m}_{w}^{2}+f \cdot \dot{m}_{w}+g \\
a=2.45 \cdot 10^{-15} \quad b=-1.1 \cdot 10^{-11} \quad c=1.9 \cdot 10^{-9} \\
d=-1.52 \cdot 10^{-5} \quad e=0.0046 \quad f=1.17 \quad g=26.62
\end{array}\right.
$$

It is to note that the Transcritical $\mathrm{CO}_{2}$ cycle has a minimum cycle temperature of $15^{\circ} \mathrm{C}$ in order to enable condensation [14]. Hence, the water temperature at cooler inlet in this cycle is set to $10^{\circ} \mathrm{C}$ wheras the range $\Delta T_{\text {range }}$ remains the same as in the other cycles.

\section{Balance of Plant}

Balance of Plant $(B o P)$ is the last contribution to the total installed cost. This cost $C_{t o t, B o P}$ is assumed to be in the range from 10 to $20 \%$ of the total installated cost. Other owner's costs or costs related to engineering, procurement and construction are not included in the calculations.

\section{Dependence on Cycle Pressure Ratio}

Figures 7 and 8 present the Overnight Capital Cost $(O C C)$ and the Power Block cost as a function of the maximum cycle pressure for values up to $40 \mathrm{MPa}$ (the last black marker in Fig. 1 corresponds to a maximum pressure of $40 \mathrm{MPa}$ ). All cycles exibit similar features. The Ovenight Capital Cost decreases as the peak pressure gets higher and then, once a minimum $O C C$ is achieved, it rises again as pressure continues to grow; the initial decay is mostly thanks to a higher thermal efficiency, see Fig. 1, whilst the subsequent rise is due to the very high operating pressures. The Power Block cost presents a somewhat mirrored trend, with highest costs at very high pressures and also high costs when pressure is very low. In this case, it is also observed that the pressure at which the PB cost is lowest depends on on cycle configuration. This becomes particularly evident for the Schroder-Turner and Precompression cycles which reach very high PB costs (higher than 100 $\mathrm{M} \$$ ) for pressures around $40 \mathrm{MPa}$ due to two counteracting effects. At low to intermediate pressures, the rise of thermal efficiency is dominant and brings about a PB cost reduction. On the other hand, at high pressure, the equipment get bulkier to endure the extremely high mechanical stresses, and this has a large impact on the cost of major components. Generally speaking, almost all the configurations exhibit a minimum $P B$ and $O C C$ at around $25 \mathrm{MPa}$ and 30-35 MPa respectively.

Based on the information Figures 7 and 8 the authors' assumption to consider a 30 MPa peak pressure for the reference power plant is validated. Among the feasible pressures, ether mechanically or thermodynamicall, this value ensures a significant reduction of the Overnight Capital cost from which it can be inferred that the PB cost has a smaller effect on OCC compared with other subsystems, in particular the solar field and thermal energy storage system. 


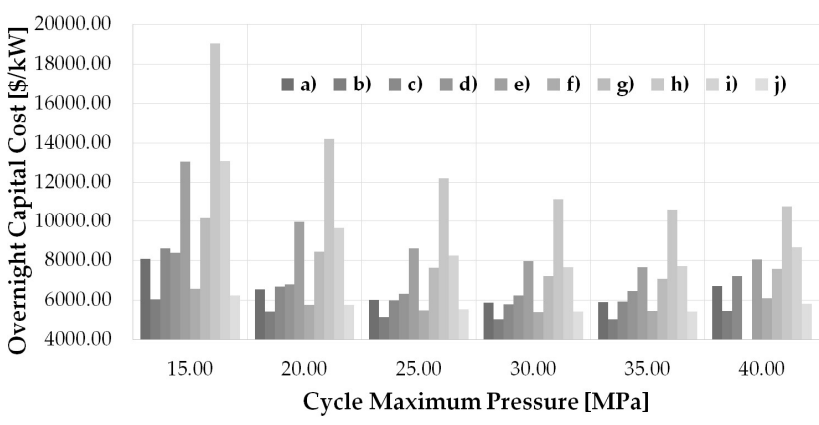

Fig. 7. Overnight Capital Costs per kilowatt as a function of power cycle maximum pressure. All cycles (see Fig. 2 to identify labels).

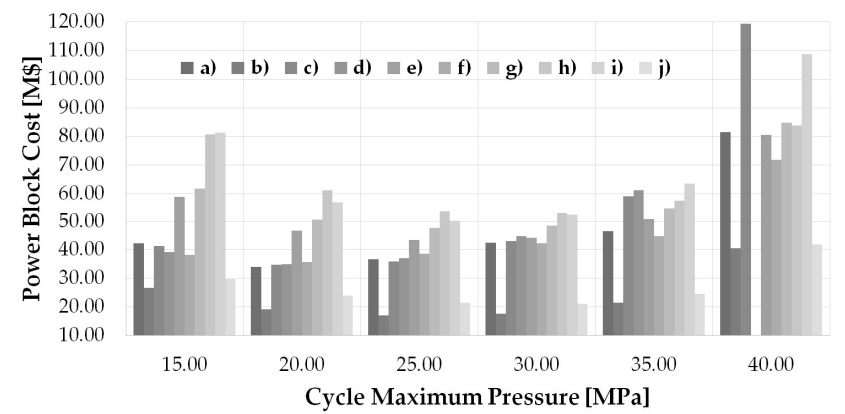

Fig. 8. Power Block Costs as a function of power cycle maximum pressure. All cycles (see Fig. 2 to identify labels).

\section{UNCERTAINTY ANALYSIS}

As it is common to any cost analysis [28], the foregoing discussion on how to estimate the costs of the different components in a supercritical $\mathrm{CO}_{2}$ power plant holds an inevitable degree of uncertainty. In order to address it, a probabilistic approach to uncertainty quantification based on the Montecarlo methodology is incorporated into the assessment. This tool is highly recommended to take into account the uncertainty encountered in the cost estimation process, especially if an innovative technology like $\mathrm{sCO}_{2}$ is considered.

Based on a similar analysis by Ho et al. [29,30], uniform probability distributions are assigned to each main parameter in the cost functions presented before. This means constant probability between the maximum and minimum values that each parameter can take, as reported in Table 2. The limits are wider for those parameters which bring in larger uncertainty whereas the range is lower for the most mature technology, for instance the solar field. With these boundary conditions, and setting the number of samples to 10000 , the Overnight Capital Cost (OCC) is calculated.

The uniform probability distribution used in $[29,30]$ has been selected for its capacity to define the boundary conditions more clearly and for its low computational duty ${ }^{2}$. The authors of this paper have also performed the calculations with a normal distribution in order to double check potential errors (larger uncertainty) coming from the selection of a wrong probability function. The results obtained with both distributions are almost identical, but with a significant computational cost saving for the uniform distribution, which is used in the remainder of this work.

For the turbomachinery in Table 2, the uniform distribution considers that a 40\% higher cost than in Eqs.(4-9) is possible, whilst the possibility to have a lower cost is considered negligible. Something similar applies to the cost of the cooling tower

\footnotetext{
${ }^{2}$ Personal communication with Craig Turchi, NREL.
} 


\begin{tabular}{cccc}
\hline \hline Cost [k\$] & Min Value & Max Value & Distribution \\
\hline Compressor & $C_{\text {compr }}$ & $1.4 \cdot C_{\text {compr }}$ & Uniform \\
Pump & $C_{\text {pump }}$ & $1.4 \cdot C_{\text {pump }}$ & Uniform \\
Turbine & $C_{\text {turb }}$ & $1.4 \cdot C_{\text {turb }}$ & Uniform \\
SS 316L [\$/kg] & 20 & 25 & Uniform \\
Inconel 617 [\$/kg] & 120 & 180 & Uniform \\
Cooling Tower & $0.7 \cdot C_{C T}$ & $1.3 \cdot C_{C T}$ & Uniform \\
Solar Field & $C_{S F}$ & $1.1 \cdot C_{S F}$ & Uniform \\
TES & $0.8 \cdot C_{T E S}$ & $1.3 \cdot C_{T E S}$ & Uniform \\
Receiver & $1.3 C_{R}$ & $1.8 \cdot C_{R}$ & Uniform \\
Tower & $C_{t o w e r}$ & $1.1 \cdot C_{t o w e r}$ & Uniform \\
BoP & $0.1 \cdot C_{t o t}$ & $0.2 \cdot C_{t o t}$ & Uniform \\
\hline \hline
\end{tabular}

Table 2. Uncertainty analysis. Limits of the uniform probability distributions.

for which potential changes of $\pm 30 \%$ are foreseen due to large variations in relative humidity at the selected plant site. The solar field is already a mature technology and thus only a 10\% deviation towards higher costs is considered, possibly brought about by unforeseen local constraints. The range shown in Table 2 for the thermal energy storage system is wider, accounting for potential changes between $-20 \%$ and $+30 \%$ with respect to the cost estimated by the in-house code, Fig. 4 . This range is intended to leave room for a new generation of less costly molten salts being introduced in the market in the near to mid future. Finally, the cost of the receiver $C_{R}$ is thought to potentially be up to $50 \%$ higher than the value provided by Fig. 3 , due to unforeseen material-related issues encountered when operating the system at very high temperatures.

\section{RESULTS}

This section presents the results provided by the uncertainty analysis using the Montecarlo method. To this aim, the probability density functions of the $O C C$-per- $k W$ are presented first, providing an overall scenario of the configurations considered. Secondly, a comparison between these configurations is developed, considering the $85 \%$ percentile and analysing the individual contributions to $O C C$.

The results of this analysis are presented in Table 3. The information shown contains the main performance parameters $\left(\eta_{t h}, W_{s}, \Delta T_{\text {solar }}\right)$ and the compressor inlet pressure and temperature considered in each case.

\section{Probability Density Functions}

The Overnight Capital Costs per installed electric kilowatt of the cycles presented in Fig. 2 are presented in Fig. $9^{3}$. Three regions are identified in this chart. On the right hand side, the Double Reheated cycle is identified as having the highest $O C C$-per-kW with up to $14000 \$ / \mathrm{kW}$. In the central region, the Recompression $+I C+R H, S c h r o d e r-T u r n e r$ and Partial Cooling $+R H$ cycles exhibit high $O C C$-per-kW with up to $10000 \$ / \mathrm{kW}$. Finally, the other six layouts are on the left hand side of the chart with installed costs in the range from 5000 to $7000 \$ / \mathrm{kW}$.

\footnotetext{
${ }^{3}$ Note that the Quasi Combined layout is excluded for its low Second Law efficiency brought about by the cryogenic cooling, as observed in Fig. $1 \mathrm{~b}$. GTP-18-1741, Sánchez, Page 14 Copyright $\odot 2018$ by ASME
} 


\begin{tabular}{cccccc}
\hline \hline Cycle & $\begin{array}{c}T_{\text {in,compr }} \\
{\left[{ }^{\circ} \mathrm{C}\right]}\end{array}$ & $\begin{array}{c}P_{\text {in,compr }} \\
{[\mathrm{MPa}]}\end{array}$ & $\begin{array}{c}\eta_{\text {th }} \\
{[\%]}\end{array}$ & $\begin{array}{c}W_{s} \\
{[\mathrm{~kJ} / \mathrm{kg}]}\end{array}$ & $\begin{array}{c}\Delta T_{\text {solar }} \\
{\left[{ }^{\circ} \mathrm{C}\right]}\end{array}$ \\
\hline $\mathrm{a}$ & 32 & 7.5 & 45.8 & 171 & 290 \\
$\mathrm{~b}$ & 15 & 5 & 48.3 & 242 & 290 \\
$\mathrm{c}$ & 32 & 7.5 & 50.6 & 164 & 254 \\
$\mathrm{~d}$ & 32 & 7.5 & 50.5 & 142 & 220 \\
$\mathrm{e}$ & 32 & 7.5 & 52.8 & 174 & 130 \\
$\mathrm{f}$ & 32 & 5 & 51.1 & 192 & 290 \\
$\mathrm{~g}$ & 32 & 5 & 53.0 & 210 & 157 \\
$\mathrm{~h}$ & 32 & 5 & 52.8 & 159 & 80 \\
$\mathrm{i}$ & 32 & 7.5 & 49.0 & 200 & 160 \\
$\mathrm{j}$ & 32 & 3 & 45.0 & 252 & 290 \\
\hline \hline
\end{tabular}

Table 3. Parameters used in the economic analysis.

The first interesting observation is related to the impact of uncertainty. It is worth noting that those cycles with higher costs also experience larger uncertainty, manifested as a less steep slope which broadens the range of possible installed cost values. For instance, for the Double Reheated layout, this range increases to more than $4000 \$ / \mathrm{kW}$ between the least and most probable costs. This is symptomatic of a larger data dispersion in the Montecarlo simulation, due to the higher relative importance of the thermal energy storage system and the tower/receiver. Such behaviour could have been deduced from the values given in Table 2 for the latter component.

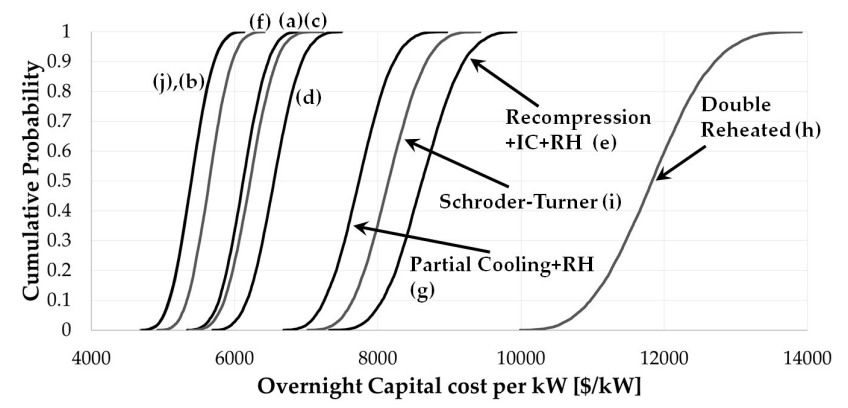

Fig. 9. Cumulative probability distribution of Overnight Capital Costs per kilowatt. All cycles (see Fig. 2 to identify labels).

The physical explanation of the foregoing discussion has to do with the fact that these cycles with higher costs are extremely recuperative, leading to significantly smaller values of $\Delta T_{\text {solar }}$ (smaller temperature rise in the heaters) and an exponential rise of the size of receiver and TES. On the other end, heat recovery in the Transcritical $\mathrm{CO}_{2}$ layout is not particularly enhanced, yielding a larger $\Delta T_{\text {solar }}$ and a more vertical slope in Fig. 9. This is also observed in the close-up of those cycles with lowest capital cost presented in Fig. 10. It is easily concluded in this latter figure that the Transcritical $\mathrm{CO}_{2}$ and Allam cycles are the only layouts likely to yield an OCC lower than $6000 \$ / \mathrm{kW}$ whilst the Partial Cooling cycle yields the same value but with $90 \%$ confidence only. 


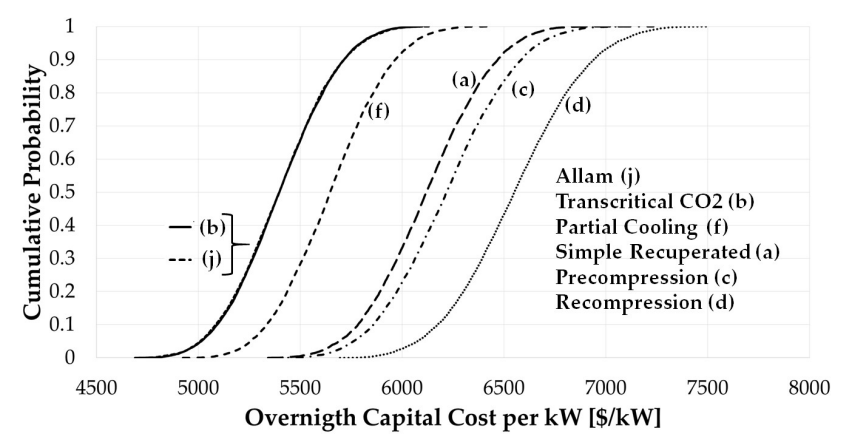

Fig. 10. Cumulative probability distribution of Overnight Capital Costs per kilowatt. Close-up of Fig. 9 .

\section{Capital Cost Comparison}

Upon evaluation of the impact of uncertainty, the $85 \%$ percentiles are used to perform a capital cost comparison of the ten cycles considered in this work. The comparison is presented in Fig. 11 with the labels already presented in Fig. 2.

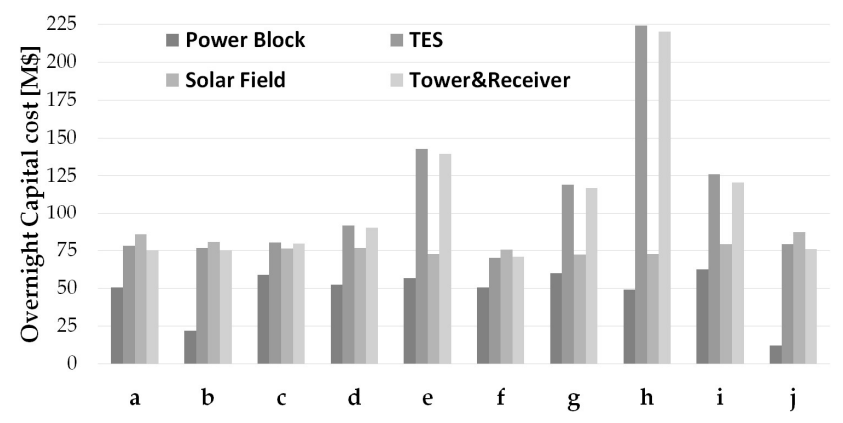

Fig. 11. Breakdown of Capital Costs. Labels refer to Fig. 2.

At first glance, the Recompression $+I C+R H$ and Double Reheated layouts ( $e$ and $h$ in Fig. 11) exhibit unusual results. Indeed, the costs of thermal energy storage and tower/receiver are significantly higher than the solar field which is usually the most expensive subsystem in a CSP plant. A similar though slightly attenuated pattern is presented by other layouts: Recompression, Schroder-Turner and Partial Cooling $+R H\left(d, i\right.$ and $g$ respectively). This is due to the very low $\Delta T_{\text {solar }}$, which is actually much lower than $\Delta T_{r e f}$ and leads to a dramatic increase in the size of these components. In particular, the Double Reheated layout presents a $\Delta T_{\text {solar }}$ of $80^{\circ} \mathrm{C}$, a value three times lower than $\Delta T_{r e f}\left(284^{\circ} \mathrm{C}\right)$ and the maximum $\Delta T_{\text {solar }}$ achieved by some of the configurations considered $\left(290^{\circ} \mathrm{C}\right.$, see Table 3$)$. The conclusion already reported in [15] with regards to the capital importance of $\Delta T_{\text {solar }}$ is confirmed again here.

Another interesting observation in Fig. 11 is the share of the solar field in those cycles with more complex layouts $(e, g$, $h, i)$ which is indeed lower thanks to a higher efficiency $\eta_{t h}$. Unfortunately, this lower cost is outweighed by the much higher cost of the remaining components in the plant. It is also worth noting that the cost of the power block is significantly lower than that of the solar subsystem in those cycles characterised by simpler configurations ( $b$ and $j$, for instance). On the other hand, the costs of power block and solar field are comparable in those cycles incorporating reheating and intercooling $(e, g)$, due to the higher thermal efficiencies achieved. Nevertheless, on the negative side, these cycles typically exhibit low $\Delta T_{\text {solar }}$, 
see Table 3, which leads to extremely high $C_{T E S}, C_{R}$ and $C_{\text {tower }}$. This can be better observed in Fig. 12 where a breakdown of the power block cost is provided.

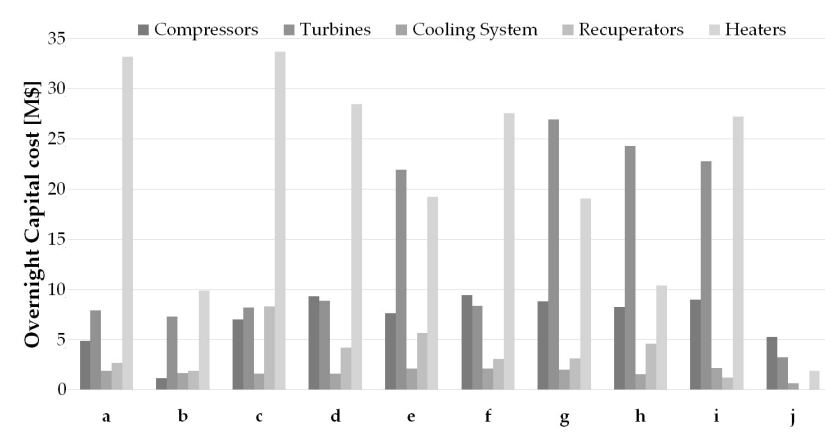

Fig. 12. Breakdown of Power Block Costs. Labels refer to Fig. 2.

The Recompression $+I C+R H$, Partial Cooling $+R H$, Double Reheated and Schroder-Turner layouts in Fig. $12(e, g$, $h$ and $i$ ) show a high turbine cost, due to the larger number of turbomachineries required by the reheating configuraton. Nevertheless, the costliest items turn out to be the heaters, owing to the more expensive materials that must be used to withstand the extremely high temperature at the inlet. Furthermore, the cost of the heaters is directly affected by $\Delta T_{\text {solar }}$ given that, for a given output, a larger temperature rise across the heater implies a lower $\Delta T$ across the solar receiver. This also implies a smaller temperature difference between the hot and cold tanks of the thermal energy sorage system. This can be inferred from the paralell trends of TES and heaters costs, yellow bars in Figs. 11 and 12 respectively.

The thermodynamic information presented by the authors in [14] and the cost analysis in this paper are integrated in Fig. 13. This chart presents a comparison between the ten cycles considered in terms of $1^{\text {st }}$ and $2^{\text {nd }}$ Law efficiencies and $O C C$, allowing to better understand the foregoing discussion. It is easily observed that the Transcritical $\mathrm{CO}_{2}(\mathrm{~b}), \mathrm{Allam}$ $(j)$ and Partial Cooling $(f)$ cycles are the least expensive options. Nevertheless, while the first two configurations do not exhibit particularly good thermodynamic features, the Partial Cooling system seems to provide a better compromise. This is further assessed in Fig. 14, where the trade-offs between the key figures of merit of each cycle are presented. These metrics are thermal efficiency $\eta_{t h}$, Carnot Factor $C F$, temperature rise across the receiver $\Delta T_{\text {solar }}$ and installed cost (expressed as $1-\$ / \mathrm{kW})$.

Thermal efficiency has a direct impact on the size of the solar field and, accordingly, the tower and receiver. The Carnot Factor is a measure of the overall irreversibility of the cycle, hence the temperature gap (between the hot and cold reservoirs) needed to achieve a given thermal efficiency; i.e., a combination of thermal efficiency and compressor inlet temperature for cycles operating at constant turbine inlet temperature. The temperature rise is an indirect measure of the inventory of molten salts that is needed to run the cycle and store thermal energy in the Thermal Energy Storage system. Finally, the complementary relative cost $1-\$ / \mathrm{kW}$ speaks for itself.

The aim of Fig. 14 is to provide a graphical comparison of the overall performance of the cycles, both thermally and economically. Accordingly, the layout achieving highest value in each axis (note that each metric is expressed in relative 


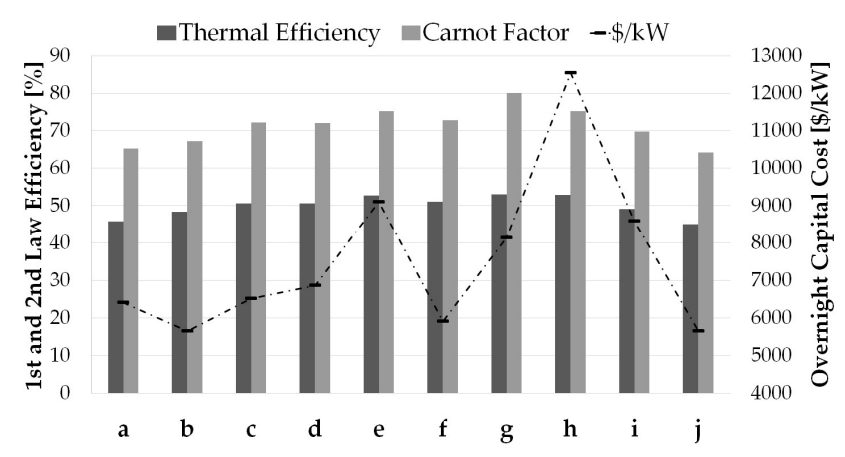

Fig. 13. Thermo-economic comparison of supercritical $\mathrm{CO}_{2}$ cycles.

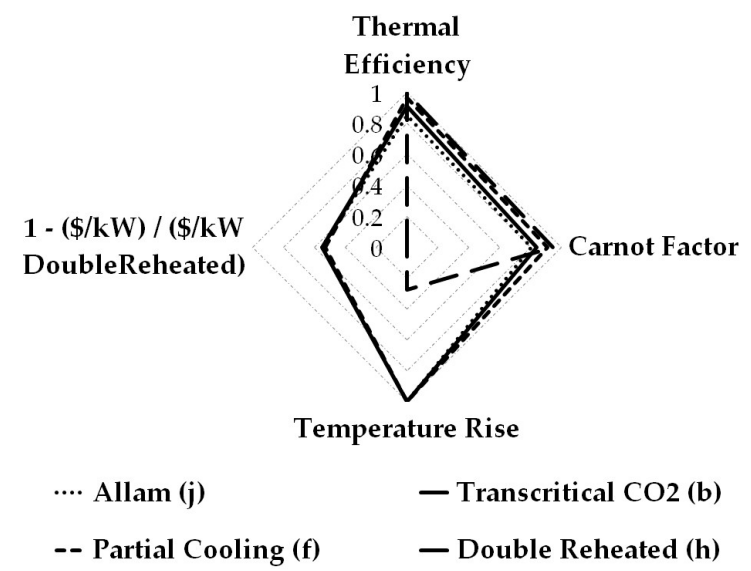

Fig. 14. Thermo-economic comparison of supercritical $\mathrm{CO}_{2}$ cycles. Trade-offs between key figures of merit.

terms for the sake of the comparison) stems as the best option since it provides highest production of energy at the minimum cost. With this in mind, it becomes clear that the Double Reheated layout cannot be considered the best choice because it exhibits an extremely high $O C C(1-\$ / \mathrm{kW} \rightarrow 0)$ in spite of its high thermal efficiency. The other three cycles, on the other hand, present very similar areas.

The uncertainty analysis economic results (Fig. 10) suggest that either the Transcritical $\mathrm{CO}_{2}$ or the Allam cycles would be the layouts of choice for the CSP application considered. The Partial Cooling cycle presents on the other hand significantly higher thermal efficiency and Carnot factor, as shown in Fig. 13, with a slightly higher $\$ / \mathrm{kW}$ (Fig. 14). For these reasons, the Partial Cooling cycle would step forth as a shorter-term, slightly more feasible option whereby a balanced techno-economic performance would be attained with less demanding design constraints for the solar receiver. On the negative side, this would be at the cost of a larger inventory of salts as shown in Fig. 14.

\section{CONCLUSIONS}

This paper presented an assessment of the Overnight Capital Cost of a $50 \mathrm{MW}_{e}$ CSP power plant with a 10 hour Thermal Energy Storage system, operating at high temperature and employing a sCO $\mathrm{SO}_{2}$ power cycle. The major equipment of the plant have been modelled either with validated in-house codes (Thermal Energy Storage, heat exchangers) or using software accepted by the industry (SAM for the solar field, tower and receiver, and Thermoflex for the turbomachinery and cooling 
tower). The commercial software has been employed to calculate reference costs of a steam-based CSP plant with a TES of similar capacity using state-of-the-art molten salts. Then, a series of correction factors have been developed in order to account for the difference between the high temperature salt FLiNaK and the reference salt, thus adapting the cost estimates to plants based on $\mathrm{SCO}_{2}$ technology.

The integral thermo-economic analysis applied to the cycles already explored by the authors in a previous work has been based on the Overnight Capital Cost per kilowatt and on efficiency according to the $1^{\text {st }}$ and $2^{\text {nd }}$ Laws of Thermoydnamics. A first conclusion is that only the Transcritical $\mathrm{CO}_{2}$ and Allam cycles seem to be likely to enable installation costs lower than $6000 \$ / \mathrm{kW}$ with a $100 \%$ probability. If the $85 \%$ confidence interval is considered, the capital costs of these two cycles are 5657 and $5655 \$ / \mathrm{kW}$ respectively, which seems to be competitive against some $3800 \$ / \mathrm{kW}$ for a coal power plant [31] or $5800 \$ / \mathrm{kW}$ for a state-of-the-art CSP plant using tower technology [32]. Interestingly, none of these configurations present a remarkably high $\eta_{t h}$ (lower than $48.5 \%$ ) or a very high Carnot Factor.

The Partial Cooling layout follows close behind with $5907 \$ / \mathrm{kW}$, combined in this case with very good thermodynamic features. This cycle provides a thermal efficiency higher than 51\%, and apparently the best compromise between thermodynamic and economic features. Finally, very complex layouts seem to be not advisable, even if they are characterised by really high thermal efficiencies $\eta_{t h}$. The Double Reheating, Recompression $+I C+R H$ and Partial Cooling $+R H$ cycles are actually able to exceed $53 \%$ thermal efficiency but suffer from a much larger number of components, some of them operating at high temperature. As a consequence, their capital costs per kilowatt increase to 12538, 9096 and 8130 \$/kW respectively.

\section{ACKNOWLEDGMENT}

The authors are grateful to Dr. Craig Turchi of the National Renewable Energy Laboratory for the very interesting discussion about the best probability distributions for the Montecarlo methodology.

\section{References}

[1] Turchi, C. S., Ma, Z., Neises, T. W., and Wagner, M. J., 2013. “Thermodynamic Study of Advanced Supercritical Carbon Dioxide Power Cycles for Concentrating Solar Power Systems”. Journal of Solar Energy Engineering, 135(4), p. 041007.

[2] Wang, K., He, Y.-L., and Zhu, H.-H., 2017. "Integration Between Supercritical $\mathrm{CO}_{2}$ Brayton Cycles and Molten Salt Solar Power Towers: A Review and A Comprehensive Comparison of Different Cycle Layouts". Applied Energy, 195, pp. 819-836.

[3] Crespi, F., Gavagnin, G., Sánchez, D., and Martínez, G. S., 2017. “Supercritical Carbon Dioxide Cycles for Power Generation: A Review". Applied Energy, 195, pp. 152-183.

[4] Dostal, V., Driscoll, M. J., and Hejzlar, P., 2004. A Supercritical Carbon Dioxide Cycle for Next Generation Nuclear Reactors. Phd Thesis MIT-ANP-TR-100, Massachusetts Institute of Technology, Cambridge, MA.

[5] Dennis, R., 2017. “Overview of Supercritical Carbon Dioxide Based Power Cycles for Stationary Power Generation”. In IV International Seminar on ORC Power Systems, ORC2017. 
[6] Wright, S. A., Davidson, C. S., and Scammell, W. O., 2016. "Thermo-Economic Analysis of Four $\mathrm{sCO}_{2} \mathrm{Waste}^{\mathrm{Heat}}$ Recovery Power Systems”. In $5^{\text {th }}$ International Supercritical $\mathrm{CO}_{2}$ Power Cycle Symposium, Vol. 2, pp. 28-31.

[7] Driscoll, M. J., 2004. Supercritical $\mathrm{CO}_{2}$ Plant Cost Assessment. Report MIT-GFR-019, Masachussets Institute of Technology, Cambridge, MA.

[8] De Barbadillo, J., Baker, B. A., and Gollihue, R., 2011. "Nickel-Base Superalloys for Advanced Power Systems: An Alloy Producer's Perspective". In Proceeding of the 4th Symposium on Heat Resistant Steels and Alloys for High Efficiency USC Power Plants, China.

[9] Cich, S., Moore, J., Rimpel, A., and Hoopes, K., 2016. "Supercritical $\mathrm{CO}_{2}$ Power Cycle Limits Based on Material Cost". In The $5^{\text {th }}$ Supercritical $\mathrm{CO}_{2}$ Power Cycles Symposium.

[10] Hinze, J. F., Nellis, G. F., and Anderson, M. H., 2017. “Cost Comparison of Printed Circuit Heat Exchanger to Low Cost Periodic Flow Regenerator for Use as Recuperator in a $\mathrm{sCO}_{2}$ Brayton Cycle”. Applied Energy.

[11] Kim, I. H., Zhang, X., Christensen, R., and Sun, X., 2016. "Design Study and Cost Assessment of Straight, Zigzag, S-shape, and OSF PCHEs for a FLiNaK-sCO $\mathrm{SO}_{2}$ Secondary Heat Exchanger in FHRs". Annals of Nuclear Energy, 94, pp. 129-137.

[12] National Renewable Energy Laboratory, Solar Advisor Model Reference Manual for CSP Trough Systems, NREL (2009).

[13] Schmitt, J., Wilkes, J., Allison, T., Bennett, J., Wygant, K., and Pelton, R., 2017. "Lowering the Levelized Cost of Electricity of a Concentrating Solar Power Tower with a Supercritical Carbon Dioxide Power Cycle". In ASME Turbo Expo 2017: Turbomachinery Technical Conference and Exposition, American Society of Mechanical Engineers, pp. V009T38A028-V009T38A028.

[14] Crespi, F., Gavagnin, G., Sánchez, D., and Martínez, G. S., 2017. "Analysis of the Thermodynamic Potential of Supercritical Carbon Dioxide Cycles: A Systematic Approach”. Journal of Engineering for Gas Turbines and Power, 140(5), p. 051701.

[15] Crespi, F., Sánchez, D., Rodríguez, J. M., and Gavagnin, G., 2017. "Fundamental Thermo-Economic Approach to Selecting sco2 Power Cycles for CSP Applications”. Energy Procedia, 129, pp. 963-970.

[16] Binotti, M., Astolfi, M., Campanari, S., Manzolini, G., and Silva, P., 2017. "Preliminary Assessment of sCO $\mathrm{Cycles}^{2}$ for Power Generation in CSP Solar Tower Plants". Applied Energy.

[17] Padilla, R. V., Too, Y. C. S., Benito, R., and Stein, W., 2015. "Exergetic Analysis of Supercritical $\mathrm{CO}_{2} \mathrm{Brayton}^{\mathrm{Cycles}}$ Integrated with Solar Central Receivers”. Applied Energy, 148, pp. 348-365.

[18] Martín, M., and Sánchez, D., 2018. “A Detailed Techno-Economic Analysis of Gas Turbines Applied to CSP Power Plants with Central Receiver”. In ASME Turbo Expo 2018: Turbomachinery Technical Conference and Exposition, American Society of Mechanical Engineers.

[19] Romatoski, R., and Hu, L., 2017. "Fluoride Salt Coolant Properties for Nuclear Reactor Applications: A Review". Annals of Nuclear Energy, 109, pp. 635-647.

[20] Rodríguez, J. M., Sánchez, D., Martínez, G. S., Ikken, B., et al., 2016. “Techno-Economic Assessment of Thermal 
Energy Storage Solutions for a 1MWe CSP-ORC Power Plant”. Solar Energy, 140, pp. 206-218.

[21] Hoopes, K., Sánchez, D., and Crespi, F., 2016. “A New Method for Modelling Off-Design Performance of sCO $\mathrm{CH}_{2}$ Exchangers Without Specifying Detailed Geometry". In The $5^{\text {th }}$ Supercritical $\mathrm{CO}_{2}$ Power Cycles Symposium.

[22] Crespi, F., Gavagnin, G., Sánchez, D., and Martínez, G. S., 2017. “The Conductance Ratio Method for Off-Design Heat Exchanger Modeling and its Impact on an $\mathrm{sCO}_{2}$ Recompression Cycle”. In ASME Turbo Expo 2017: Turbomachinery Technical Conference and Exposition, American Society of Mechanical Engineers, p. V009T38A025.

[23] Yoon, S.-J., Sabharwall, P., and Kim, E.-S., 2013. Analytical study on thermal and mechanical design of printed circuit heat exchanger. Tech. rep., Technical report, Idaho Nation Laboratory, Idaho Falls, 83415.

[24] Cis Inspector, 30/11/2018, https://www.cis-inspector.com/asme-code-calculation-allowable-stresses-high-alloy.html.

[25] Special Metals, 30/11/2018, http://www.specialmetals.com/assets/smc/documents/alloys/inconel/inconel-alloy617.pdf.

[26] Couper, J. R., Penney, W. R., and Fair, J. R., 2009. Chemical Process Equipment-Selection and Design (Revised 2nd Edition). Gulf Professional Publishing.

[27] Thermoflow Inc., 2 Willow St., Suite 100, Southborough, MA 01745-1020, USA, http://www.thermoflow.com.

[28] Gavagnin, G., Sánchez, D., Martínez, G. S., Rodríguez, J. M., and Muñoz, A., 2017. “Cost Analysis of Solar Thermal Power Generators Based on Parabolic Dish and Micro Gas Turbine: Manufacturing, Transportation and Installation". Applied Energy, 194, pp. 108-122.

[29] Ho, C. K., and Kolb, G. J., 2010. “Incorporating Uncertainty into Probabilistic Performance Models of Concentrating Solar Power Plants". Journal of Solar Energy Engineering, 132(3), p. 031012.

[30] Ho, C., Mehos, M., Turchi, C., and Wagner, M., 2014. "Probabilistic Analysis of Power Tower Systems to Achieve Sunshot Goals". Energy Procedia, 49, pp. 1410-1419.

[31] Various, 2012. Cost and Performance Data for Power Generation Technologies. Tech. rep., Black \& Veatch for the National Renewable Energy Laboratory, Cambridge, MA.

[32] Various, 2016. The Power to Change: Solar and Wind Cost Reduction Potential to 2025. Tech. rep., IRENA, Abu Dhabi, United Arab Emirates. 